\title{
ALGORITHM MODELING FOR CONSTRUCTING A CONCAVE EFFICIENT FRONTIER
}

\author{
Juliana Benicio $^{1}$ and João Carlos Soares de Mello ${ }^{2}$
}

Received July 15, 2019 / Accepted March 3, 2020

\begin{abstract}
When analyzed from the perspective of one input and one output, the classic Data Envelopment Analysis (DEA) model (known as BCC after its developers Banker, Charnes, and Cooper) presents an efficient frontier with "downward" concavity (convex), therefore delivering variable returns to scale. However, these returns show a decrease in marginal productivity as the number of inputs increases, that is, the frontier presents decreasing global returns to scale. Both the convex frontier (DEA BCC) and the concave frontier ("upward" concavity) present average productivities that vary along the curve; thus, the local returns to scale are variable. It is claimed that the two formats are complementary, and therefore both should be verified in the literature. Thus, this article proposes an algorithm capable of modeling an efficient frontier, for one input or one output, with increasing global returns to scale, whereby an increase in input causes an increase in marginal productivity.
\end{abstract}

Keywords: DEA, algorithm, efficient frontier, global returns to scale.

\section{INTRODUCTION}

Banker, Charnes \& Cooper (1984) developed a DEA model, known as BCC, which incorporates the economic concept of returns to scale (Banker \& Thrall, 1990). The main objective of this model is to calculate efficiencies while taking into account the productive scale efficiency in which the DMU operates (Banker et al., 2004). Therefore, the CCR proportionality condition is not guaranteed in this model, and the average productivities of the DMUs, considered as efficient, are different.

The DEA BCC model presents a "downward"-convex efficient frontier as (Benicio \& Soares de Mello, 2019). This means that:

\footnotetext{
*Corresponding author

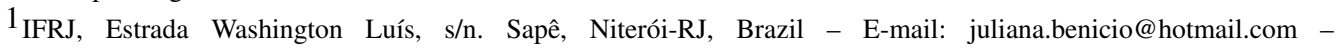
https://orcid.org/0000-0003-0976-3146

${ }^{2}$ Universidade Federal Fluminense, Niterói-RJ, Brazil - E-mail: joaocsmello@ gmail.com - http://orcid.org/ 0000-0002$6507-2721$
} 
- Average productivity varies along the frontier (Benicio \& Soares de Mello, 2015);

- Marginal productivity decreases along the DEA BCC frontier (Benicio et al., 2015).

As highlighted by Benicio \& Sores de Mello (2019), the verification of the variation of the average productivity along the frontier is adopted by most researchers, and is also known as the local returns to scale. This is because the local returns are identified immediately, and are verified in the neighborhood of a certain selected frontier point. Given this characteristic of case-by-case analysis, the local returns cannot be associated with any specific frontier format.

On the other hand, the verification of the variation of the marginal productivity along the efficient frontier can also be used, for this reason, the literature has incorporated the concept of global returns to scale (Miyazaki, 2001; Podinovski, 2004; Fried et al., 2008; Quinzii, 1992). Global returns to scale refer to cases where one single type of returns to scale are verified by the production technology (Quinzii, 1992). In such cases, as will be seen in the next section, the global returns to scale can be associated with specific frontier formats.

Podinovski (2004) has questioned the fact that the DEA literature is based on one single technology, as described by the DEA BCC frontier, to represent variable returns to scale. As highlighted by the author, the convex concavity of the DEA BCC frontier exemplifies just mechanism of variable returns to scale: that with decreasing marginal productivity (Sueyoshi, 1999); in other words, when the efficient DMU gains scale, it loses productivity. In this regard, the DEA BCC model only identifies those DMUs that have lost marginal productivity as being efficient.

The concave efficient frontier, for example, is not considered in the DEA literature and shows variable returns to scale. In a concave frontier, when the efficient DMU gains scale, it also gains productivity

Presenting a concave efficient frontier means that:

- Average productivity varies along the frontier and

- Marginal productivity increases along the efficient frontier - because of that, the frontier presents a global return to scale.

The verification of increasing marginal productivity is important because industry analysis produces scenarios in which gains with respect to scale are large and small producers, acting on a small scale, are classified as inefficient. This occurs with the DEA BCC frontier: because of the convex concavity, DMUs with scales smaller than the CCR-efficient DMU scale, which presents increasing marginal productivity, are not identified as efficient.

Therefore, the DEA BCC delimitation of a convex frontier (convexity) should not be the only procedure to model variable returns to scale. The research of new efficient frontier formats-as well as an "upward" frontier (concave concavity)—may describe different production technologies as a means of enriching the DEA literature (Podinovski, 2004). 
The objective of this article is to present an algorithm for determining efficient DMUs belonging to a concave efficient frontier. This study pursues an efficient frontier with increasing global returns to scale, called the FConc model. This feature ensures that all DMUs considered efficient by the FConc model will have increasing marginal productivity and convex efficient frontier.

\section{DEA AND VARIABLE RETURNS TO SCALE}

The original DEA CCR model (Charnes, Cooper \& Rhodes, 1978) builds a linear efficient frontier, in which it is impossible to increase any output without increasing any input or decreasing any other output (Coelli \& Perelman, 1999). Their efficiency can be compared using the following multipliers model:

$$
\begin{aligned}
& \text { Max Eff } 0=\frac{\sum_{j=1}^{s} u_{j} y_{j o}}{\sum_{i=1}^{r} v_{i} x_{i o}} \\
& \text { subject } \\
& \frac{\sum_{j=1}^{s} u_{j} y_{j k}}{\sum_{i=1}^{r} v_{i} x_{i k}} \leq 1, k=1, \ldots, n \\
& u_{j} \wedge v_{i} \geq 0 \forall j, i
\end{aligned}
$$

where $\mathbf{u}_{\mathbf{j}}, \mathbf{v}_{\mathbf{i}}$ are the output and input weights, respectively; $\mathbf{x}_{\mathbf{i k}}, \mathbf{y}_{\mathbf{j k}}$ denote inputs $\mathbf{i}$ and outputs $\mathbf{j}$ of DMU $\mathrm{K}_{\mathrm{K}}$; and $\mathbf{x}_{\mathbf{i} \mathbf{0}}, \mathbf{y}_{\mathbf{j} \mathbf{0}}$ are inputs $\mathrm{i}$ and outputs $\mathbf{j}$ of the DMU being evaluated.

The DEA BCC model creates an efficient frontier that differs from that of the CCR formulation: it is convex and the efficient DMUs need not have the same productivity (Panzar \& Willig, 1977; Baumol, Panzar \& Willig, 1982). A convex frontier presents global decreasing returns, and so the marginal productivity decreases along the entire frontier.

The DEA BCC model is based on the identification of boundaries in the multiple hyperplanes contained in a convex set of production possibilities (Thanassoulis, 2001). In this sense, considering a production of multiple inputs $(\mathrm{X})$ and outputs $(\mathrm{Y})$, we define $\mathrm{T}$ as the production possibility set, where $\{(\mathrm{X}, \mathrm{Y}) \| \mathrm{Y}$ can be produced with output $\mathrm{X}\}$.

The convexity of the production possibility set (PPS) is guaranteed by postulate 1 , which is expressed as (Banker, Charnes \& Cooper, 1984):

$$
\text { Considering that }(X, Y) \in T \wedge\left(X^{\prime}, Y^{\prime}\right) \in T \text {, then }(\lambda X+(1-\lambda) X, \lambda Y+(1-\lambda) Y) \in T ; \text { for any } \lambda \in[0,1]
$$

The PPS is then determined by:

$$
T_{B C C} \equiv\left\{(X, Y) \| X \geq \sum_{j=1}^{n} \lambda_{j} X_{j}, Y \leq \sum_{j=1}^{n} \lambda_{j} Y_{j}, \sum_{j=1}^{n} \lambda_{j}=1, \lambda_{j} \geq 0\right\}
$$

The region of viable production in the DEA BCC model is constrained by a frontier with variable returns to scale, because the DMUs present distinct productivities at different scales along this border (Senra, 2007). 
The line segment of interval $I_{x_{1} x_{2}}$ linking two points of the DEA BCC efficient frontier exhibits the characteristics of a convex frontier, and so $(1-t) f\left(x ?_{1}\right)+t f\left(x_{2}\right) \leq f\left((1-t) x_{1}+t x_{2}\right)$. Therefore, $I_{x_{1} x_{2}} \in T_{B C C}$. Thus, the BCC frontier, even though it is not differentiable, is characterized as a convex frontier.

From the convex concavity of the DEA BCC frontier and its relation with the previously presented concepts, we may conclude that efficient DMUs in this model can be analyzed according to two aspects (Benicio \& Soares de Mello, 2019):

1. Their average productivities:

The BCC-efficient DMUs present different average productivities from each other; therefore, different local returns to scale appear.

In this sense, when the model results indicate increasing returns, there has been an increase in average productivity at the analyzed point. Similarly, when the model results show decreasing returns, there has been a decrease in average productivity.

2. Their marginal productivities:

The BCC-efficient DMUs present decreasing marginal productivities along the entire frontier. In other words, a certain efficient DMU with a larger scale than another efficient one necessarily has a lower marginal productivity.

\section{BUILDING A CONCAVE EFFICIENT FRONTIER}

The purpose of this section is to present a procedure for solving problems in which the efficient frontier presents increasing global returns to scale. Therefore, along the generated efficient frontier, the marginal productivity should increase as the volume of inputs increases. This frontier will have the following main characteristics:

- Concave concavity;

- Increasing marginal productivity along the entire frontier.

As we have seen, these assumptions are in line with the concept of global variable returns to scale, in which a given DMU can be considered efficient even if it does not present the same productivity of the most productive DMU.

Knowing that:

Marginal Productivity $\left(P M_{g}\right)=\frac{\Delta f(x)}{\Delta x}$

Therefore, the global return to scale can be defined as:

- Global increasing return to scale (GIRS): $\Delta \mathrm{PMg} / \Delta \mathrm{x}>0$

- Global decreasing return to scale (GDRS): $\Delta \mathrm{PMg} / \Delta \mathrm{x}<0$ (8) 
- Global constant return to scale (GCRS): $\Delta \mathrm{PMg} / \Delta \mathrm{x}=0$

In this study, the global returns to scale are increasing.

The proposed algorithm is capable of defining efficient DMUs for one input and $n$ outputs, with the aim of producing an efficient concave frontier. We refer to the proposed method as FConc.

The FConc frontier must be constructed in such a way that a line segment joining any two efficient DMUs should not contain any points inside the set of production possibilities.

In the FConc algorithm, the marginal productivity is the main concept for determining efficient DMUs. However, the use of this index for the proposed modeling (cases of one input and $n$ outputs) becomes complex, as will be explained in the next section. For this reason, the marginal productivity is related to the CCR efficiency, making the latter a viable substitute that can be used in the algorithm.

\subsection{CCR Efficiency and Marginal Productivity}

The marginal productivity is an important measure in the concept of global returns to scale. It relates the variation of one input to the variation of one output. Thus, in some production involving $m$ inputs and $n$ outputs, $n \times m$ marginal productivities can be calculated. The proposed algorithm can be applied to cases with one input and $n$ outputs, so $n$ marginal productivities can be calculated. For this reason, the algorithm requires the definition of a multidimensional measure that represents several dimensions of the productivity analysis. The measure used in this study is the CCR efficiency, which is explained below.

The DEA efficiencies are calculated using the distance between the virtual output/virtual input ratio reached by the inefficient DMUs and the efficient frontier of the utilized model (Soares de Mello, 2008; Soares de Mello, 2005). The efficient DMUs belonging to the efficient frontier have an efficiency equal to 1 (Despotis, Stamati \& Smirlis, 2010).

DMUs with a CCR efficiency of 1 have the same average productivity and are part of the frontier. This is demonstrated in Figure 1 for one input and one output, where the efficient frontier is represented by the red line. In this example, DMU Z is the CCR-efficient DMU.

Efficiency is measured by the ratio of the productivities of efficient DMUs and inefficient DMUs. In the case of input orientation, in an example with one input and one output, the CCR efficiency measure is the ratio between the horizontal distance from the $\mathrm{y}$ axis to the efficient frontier (O'O') and the horizontal distance from the y axis to DMU O (O”O) (Cooper et al., 2001).

\subsection{FConc Algorithm for a Concave Efficient Frontier}

The FConc algorithm defines which DMUs will be considered as efficient and, therefore, be part of the efficient frontier representing global increasing returns to scale. As previously demonstrated, global increasing returns to scale occur when the increase in marginal productivity of efficient DMUs rises with increasing production scale. 


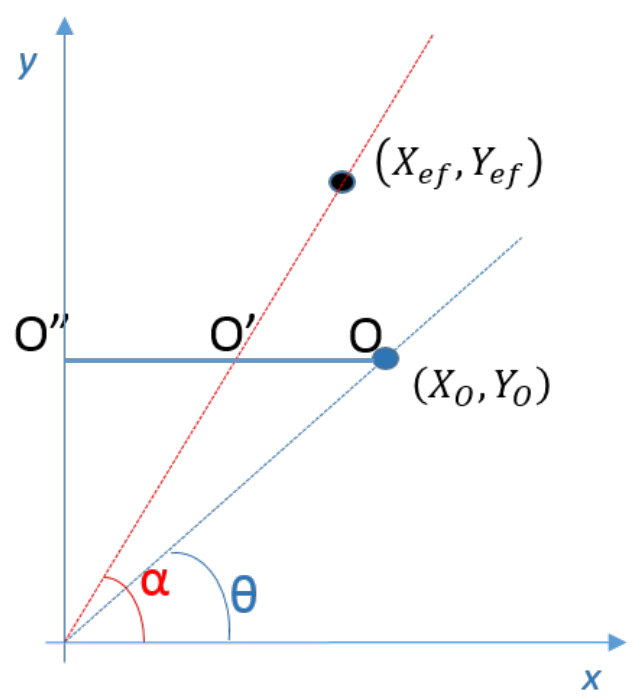

Figure 1 - Determination of CCR Efficiency.

In the FConc algorithm, to guarantee the global increasing returns to scale, we consider the efficient DMUs (Efficient CONC) to exhibit increasing CCR efficiency.

We consider the following conventions:

- DMUs are sorted, incrementally, from 1 to $m$ according to the input value;

- $E f_{C C R O}$ is the CCR efficiency of the observed DMU;

- $E f_{C C R q}$ is the CCR efficiency of all Efficient CONC DMUs preceding the observed DMU;

- DMU1 (lower input value DMU) has $E f_{C C R q}=0$.

Considering the following steps:

Step 1: SORT all analyzed DMUs by input value and CALCULATE their CCR EFFICIENCIES. In this step, the DMUs are arranged in ascending order to enable the calculation of the variation in the next step.

The DMUs are then related to their respective CCR efficiencies in association with their arrangement. The CCR efficiency replaces the marginal productivity for the selection of efficient FConc DMUs.

Step 2: CALCULATE $\Delta_{m, q}^{e f}$ such that $\Delta_{m, q}^{e f}=\left(E f_{C C R m}-E f_{C C R q}\right)$, for all $m, q$.

Such that:

- DMUs are defined as INEFFICIENT if $\Delta_{O, q}^{e f} \leq 0$ for at least one $q$. 
- DMUs are defined as EFFICIENT if $\Delta_{O, q}^{e f}>0$ for all $q$.

In this step, the variations are calculated to verify whether there was any increase in CCR efficiency. This is because, to ensure global increasing returns to scale, the CCR efficiencies must increase with the production scale. For this reason, when $\Delta_{O, q}^{e f}$ is negative, the analyzed DMU is considered inefficient. When $\Delta_{O, q}^{e f}$ is positive, the analyzed DMU is considered efficient.

In terms of applying the FConc algorithm, the following points should be noted:

- Each observed DMU will be analyzed in an orderly and increasing manner, according to the input value. Thus, the model can only be applied to one input and $\boldsymbol{n}$ outputs, as the sorting of DMUs would not be possible when working with more inputs.

- Each DMU efficiency delta ( $\Delta_{m, q}^{e f}$ ) gives the difference between the CCR efficiency of the observed DMU and all preceding Efficient CONC DMUs. Theoretically, the application of the algorithm would lead to the same result if $\Delta_{m, q}^{e f}$ was calculated between the observed DMUs and all preceding DMUs. We decided to choose only the preceding Efficient CONC DMUs as a means of accelerating the algorithm.

The results of the FConc algorithm are DMUs that belong to the efficient frontier with global increasing returns to scale. All DMUs identified as efficient will have increasing marginal productivity. The efficient frontier has concave concavity.

\section{Algorithm 1, FConc:}

Begin

1.Input: CCR efficiencies and their input values.

Output: DMUs FCon efficient

2. SORT all analyzed DMUs by input value

3. Consider DMU1 (the DMU with the lowest input value) as FCon EFFICIENT.

4. CALCULATE $\Delta_{m, q}^{e f}$ such that $\Delta_{m, q}^{e f}=\left(E f_{C C R m}-E f_{C C R q}\right)$, for all $m$, considering q the efficient DMU with the higher input value.

If $\Delta_{m, q}^{e f} \leq 0$ for at least one $m$, suchasm $\geq q$

Then DMUs are defined as FCon INEFFICIENT

Restart $m$ for $m+1$

Else $\Delta_{m, q}^{e f}>0$ for all

Then DMUs are defined as FCon EFFICIENT

Restart $\mathrm{m}$ for $m+1$

End if 
End

\subsection{Application of FConc Algorithm}

This section presents numerical examples that clarify the application of the FConc algorithm. In each of the examples, the CCR efficiency of the corresponding DMU ( $\left.\mathrm{Ef}_{\mathrm{CCR}}\right)$ was calculated using the SIAD software (Angulo Meza et al., 2005).

\subsubsection{Example 1}

To calculate the results of the FConc algorithm applied to the example 1, consider the data in Table 1, and follow the steps bellow.

Table 1 - Data of DMUs in Example 1 and CCR Efficiency results.

\begin{tabular}{|c|c|c|c|}
\hline DMU & INPUT & OUTPUT & $\begin{array}{c}\text { CCR Results } \\
\text { (Step 1) }\end{array}$ \\
\hline 1 & 0.5 & 0.5 & 0.321429 \\
\hline 2 & 1.5 & 3 & 0.642857 \\
\hline 3 & 2.5 & 6 & 0.771429 \\
\hline 4 & 3.5 & 9 & 0.826531 \\
\hline 5 & 4.5 & 14 & 1.000000 \\
\hline 6 & 6 & 18 & 0.964286 \\
\hline 7 & 7.5 & 21 & 0.900000 \\
\hline 8 & 8 & 22 & 0.883929 \\
\hline 9 & 8.5 & 22.5 & 0.850840 \\
\hline
\end{tabular}

Step 1: Calculate the CCR efficiency

DMUs 1-9 were arranged such that DMU1 had the lowest input value and DMU9 had the highest input value. Subsequently, the respective CCR efficiencies were computed.

Step 2: Calculate $\Delta_{m, q}^{e f}$

DMU 1: $\Delta_{D M U 1,0}^{e f}>0 \rightarrow$ DMU 1: Efficient.

DMU 2: $\Delta_{D M U 2,1}^{e f}>0 \rightarrow$ DMU 2: Efficient.

DMU 3: $\Delta_{D M U 3,2}^{e f}>0 ; \Delta_{D M U 31}^{e f}>0 \rightarrow$ DMU 3: Efficient.

DMU 4: $\Delta_{D M U 4,3}^{e f}>0 ; \Delta_{D M U 42}^{e f}>0 ; \Delta_{D M U 41}^{e f}>0 \rightarrow$ DMU 4: Efficient.

DMU 5: $\Delta_{D M U 5,4}^{e f}>0 ; \Delta_{D M U 53}^{e f}>0 ; \Delta_{D M U 52}^{e f}>0 ; \Delta_{D M U 51}^{e f}>0 \rightarrow$ DMU 5: Efficient.

DMU 6: $\Delta_{D M U 6,5}^{e f}<0 \rightarrow$ DMU 6: Inefficient.

DMU 7: $\Delta_{D M U 7,5}^{e f}<0 \rightarrow$ DMU 7: Inefficient. 
DMU 8: $\Delta_{D M U 8,5}^{e f}<0 \rightarrow$ DMU 8: Inefficient.

DMU 9: $\Delta_{D M U 9,5}^{e f}<0 \rightarrow$ DMU 9: Inefficient.

Solution of FConc algorithm: DMUs 6, 7, 8, and 9 are considered to be INEFFICIENT and DMUs 1, 2, 3, 4, and 5 are considered to be EFFICIENT.

Figure 2 displays a comparison between the CCR efficient frontier (green) and the concave frontier delimited by Algorithm 1 (orange) from the DMU data in Example 1.

The results given by the FConc algorithm produce a concave efficient frontier configuration, with DMUs 1-5 generating constant growth in marginal productivity (Table 2). After the CCR-efficient DMU, DMUs 6-9 produce decreasing marginal productivity, and are therefore considered inefficient.

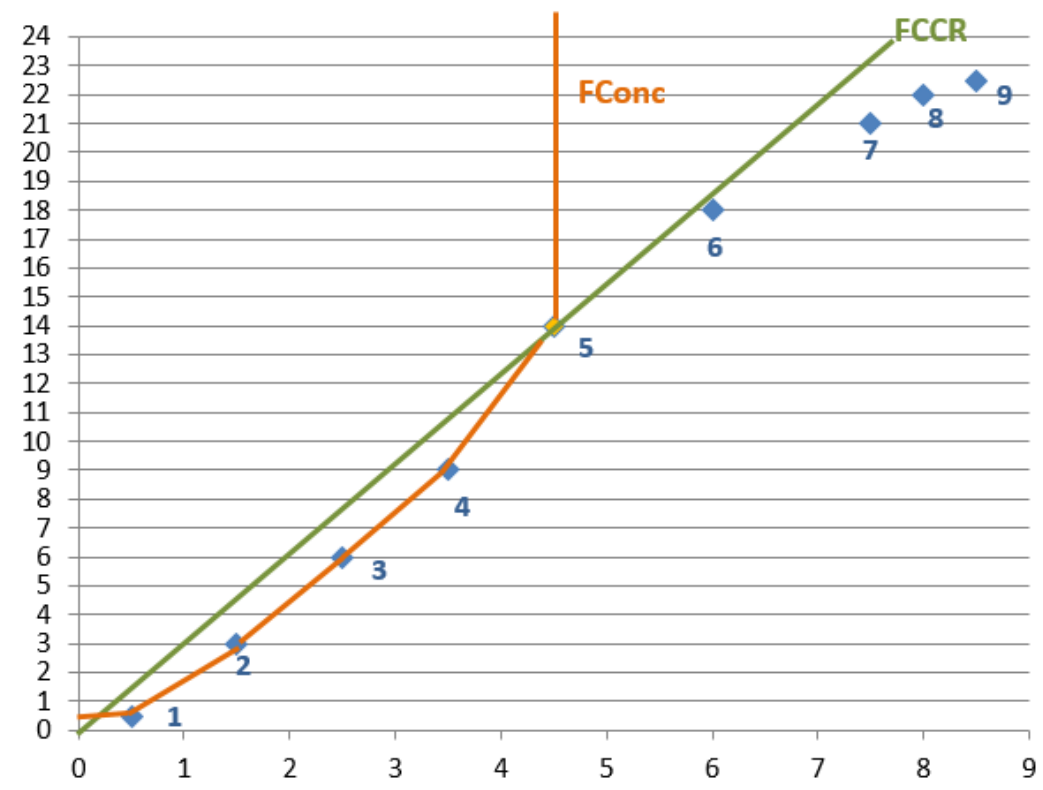

Figure 2 - FConc Frontier and CCR Frontier of Example 1.

From Figure 2, we can see that a horizontal line is generated before the first efficient DMU. This straight line respects the characteristics of the frontier with global increasing returns to scale, that is, increasing marginal productivity. If the frontier passed through the origin, the principle of increasing marginal productivity would not be guaranteed. This characteristic is analogous to the BCC model, which displays a vertical line segment before the first efficient DMU.

Note that the marginal productivities of the DMUs (Table 2) were calculated by the delta ratios between the observed DMU and the preceding efficient FConc DMU. To calculate the marginal productivity of the first DMU (that with the lowest input number), we consider an inefficient virtual DMU with zero inputs and zero outputs as a predecessor to the first observed DMU. 
Table 2 - Data of DMUs in Example 1 and their Marginal Productivities (MgP).

\begin{tabular}{|c|c|c|c|}
\hline DMU & INPUT & OUTPUT & MgP \\
\hline 1 & 0.5 & 0.5 & 1 \\
\hline 2 & 1.5 & 3 & 2.5 \\
\hline 3 & 2.5 & 6 & 3 \\
\hline 4 & 3.5 & 9 & 3 \\
\hline $\mathbf{5}$ & $\mathbf{4 . 5}$ & $\mathbf{1 4}$ & $\mathbf{5}$ \\
\hline 6 & 6 & 18 & 2.66667 \\
\hline 7 & 7.5 & 21 & 2.3333 \\
\hline 8 & 8 & 22 & 2.285 \\
\hline 9 & 8.5 & 22.5 & 2.125 \\
\hline
\end{tabular}

\subsubsection{Example 2}

To calculate the results of the FConc algorithm applied to the example 2, consider the data in Table 3, and follow the steps shown in Algorithm 1.

Table 3 - Data of DMUs in Example 2 and CCR Efficiency results.

\begin{tabular}{|c|c|c|c|}
\hline DMU & INPUT & OUTPUT & $\begin{array}{c}\text { CCR Results } \\
\text { (Step 1) }\end{array}$ \\
\hline 1 & 0,5 & 0,5 & 0,321429 \\
\hline 2 & 1 & 0,7 & 0,225000 \\
\hline 3 & 1,5 & 3 & 0,642857 \\
\hline 4 & 2,5 & 6 & 0,771429 \\
\hline 5 & 3 & 7 & 0,750000 \\
\hline 6 & 3,5 & 9 & 0,826531 \\
\hline 7 & $\mathbf{4 , 5}$ & $\mathbf{1 4}$ & 1,000000 \\
\hline 8 & 6 & 18 & 0,964286 \\
\hline 9 & 7 & 19 & 0,872449 \\
\hline 10 & 7,5 & 21 & 0,900000 \\
\hline 11 & 8 & 22 & 0,883929 \\
\hline 12 & 8,5 & 22,5 & 0,850840 \\
\hline
\end{tabular}

Solution of FConc algorithm: As DMUs 2, 5, 8, 9, 10, 11 e 12 are considered as INEFFICIENT and DMUs 1, 3, 4, 6 e 7 are considered as EFFICIENT.

From the results of Table 3, it can be seen that efficient DMUs of an efficient concave border (orange) would be DMUs: 1, 3, 4, 6 and 7; as shown in Figure 3.

The result of the FConc algorithm applied to example 2 shows the configuration of the efficient concave frontier. DMUs 1, 3, 4, 6 and 7 show constant growth of marginal productivity, see Table 


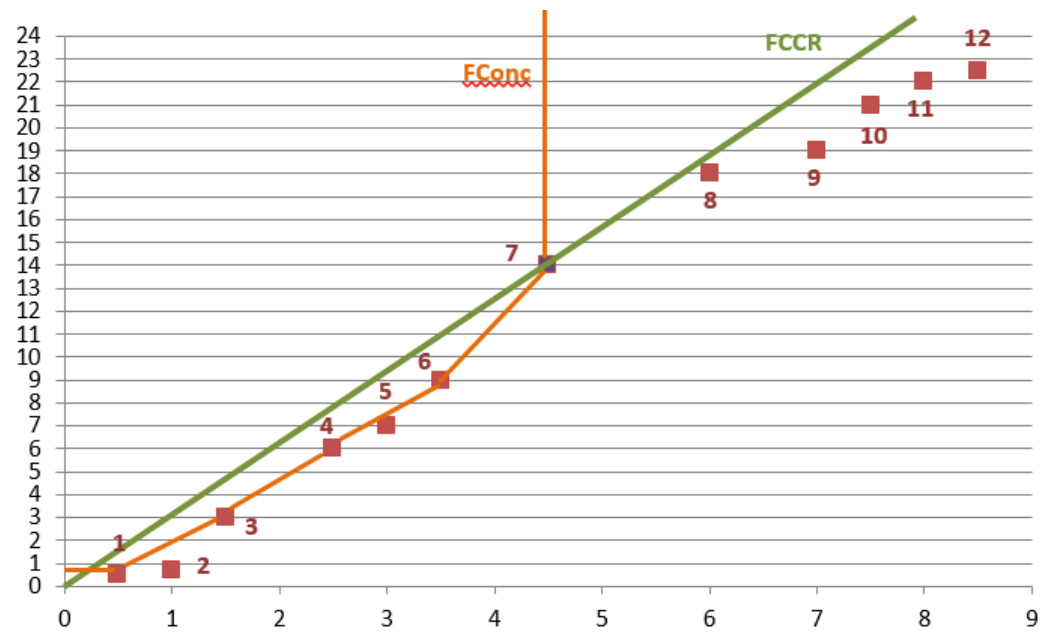

Figure 3 - FConc Frontier and Frontier CCR of Example 2.

3. After DMR CCR efficient, the DMUs presented decreasing marginal productivity, therefore, they were considered inefficient. They are: DMUs 8, 9, 10, 11 and 12.

Table 4 - Data from the DMUs of Example 2 and their Marginal Productivities.

\begin{tabular}{|c|c|c|c|}
\hline DMU & INPUT & OUTPUT & $\mathbf{M g P}$ \\
\hline 1 & 0,5 & 0,5 & 1 \\
\hline 3 & 1,5 & 3 & 2,5 \\
\hline 4 & 2,5 & 6 & 3 \\
\hline 6 & 3,5 & 9 & 3 \\
\hline $\mathbf{7}$ & $\mathbf{4 , 5}$ & $\mathbf{1 4}$ & $\mathbf{5}$ \\
\hline
\end{tabular}

It is noteworthy that DMUs 2 and 5 were considered inefficient because their marginal productivity decreases compared to the efficient DMUs that precede them. In this way, the marginal productivity of the DMUs that succeed them, DMUs 3 and 6 are calculated in comparison with the DMUs 1 and 4.

Figure 4 compares the results of efficient DMUs verified before the CCR efficient DMU of the FConc model and the DEA BCC model applied to Example 2.

It is verified that the DMU7 is the DMR CCR efficient and therefore also efficient in the BCC and FCon models. DMUs 2 and 5 are inefficient for all 3 models.

It should be noted that the BCC model considers as efficient only DMUs 1 and 7 (as well as: 8, 10, 11 and 12); for this model, DMUs 2, 3, 4, 5 and 6 are BCC inefficient. However, when analyzing the CCR result of the DMUs 3, 4 and 6, it is observed that these have increasing CCR efficiency, thus increasing marginal productivity. Thus, when considering the scales on which 


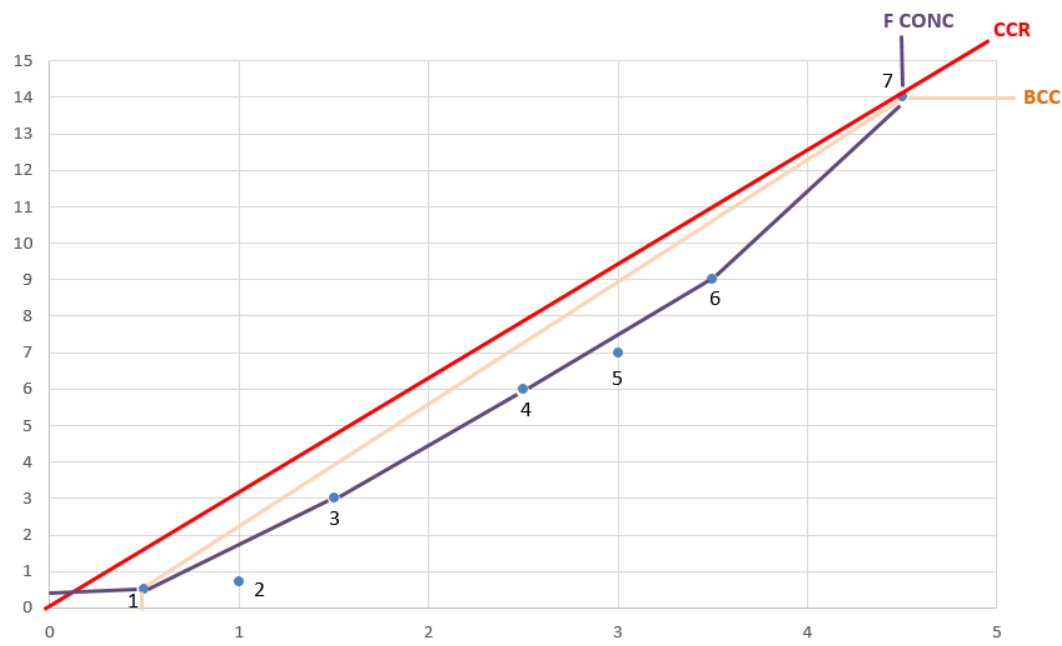

Figure 4 - Frontiers of the CCR, BCC and FConc Models of Example 2.

these DMUs act, they can be considered efficient for a model from the perspective of increasing global returns to scale, such as the FConc model.

\subsubsection{Example 3}

To calculate the results of the FConc algorithm applied to the example 3, consider the data in Table 5, and follow the steps shown in Algorithm 1.

Table 5 - Data from the DMUs of Example 3 and the results of CCR Efficiencies.

\begin{tabular}{|c|c|c|c|}
\hline DMU & INPUT & OUTPUT & $\begin{array}{c}\text { CCR Results } \\
\text { (Step 1) }\end{array}$ \\
\hline 1 & 1 & 1 & 0.33 \\
\hline 2 & 1.5 & 3.5 & 0.77 \\
\hline 3 & 2 & 6 & 1.0000 \\
\hline 4 & 2.5 & 7 & 0.93 \\
\hline 5 & 3 & 8 & 0.88 \\
\hline 6 & 4 & 9 & 0.75 \\
\hline 7 & 5 & 10 & 0.666 \\
\hline
\end{tabular}

Solution of FConc algorithm: As DMUs 4, 5, 6 e 7 are considered as INEFFICIENT and DMUs 1,2 , e 3 are considered as EFFICIENT.

From the results of Table 5, it can be seen that efficient DMUs of an efficient concave (purple) border would be DMUs: 1, 2 and 3; as shown in Figure 5. 


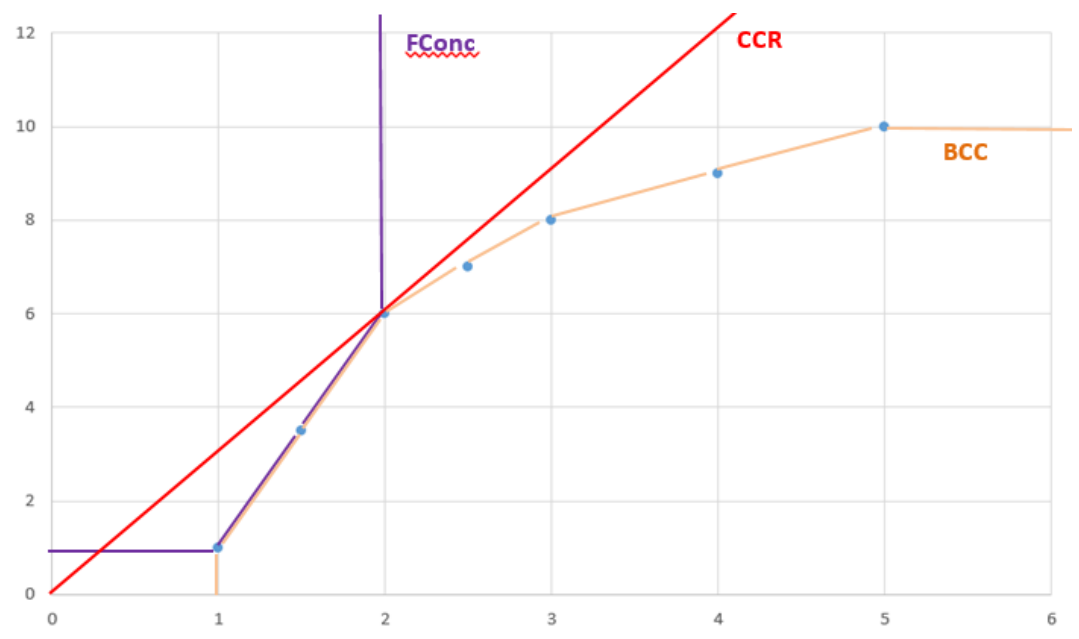

Figure 5 - Frontiers of the CCR, BCC and FConc Models of Example 3.

DMUs 1, 2 and 3 are efficient for the FConc and BCC models. However DMUs 4, 5, 6 and 7 are only BCC efficient.

It should be noted that DMUs 4, 5, 6 and 7, which were considered efficient only for the DEA BCC model, present decreasing CCR efficiency, thus decreasing marginal productivity. For this reason, they should be considered inefficient for a model from the perspective of increasing global returns to scale, such as the FConc model.

\subsubsection{Example 4: Application of the FConc Model for 1 input and 3 outputs}

In this example, the FConc model will be applied to data from one input and three outputs, accord to the Table 6 .

Table 6 - DMU data of Example 5.2 with Efficiency results according to CCR, BCC and FCONC models.

\begin{tabular}{|c|c|c|c|c|c|}
\hline DMU & INPUT & OUTPUT1 & OUTPUT2 & OUTPUT3 & Ef CCR \\
\hline 1 & 2 & 0,5 & 1 & 0,5 & 0,88 \\
\hline 2 & 3 & 1 & 1.5 & 1 & 0,87 \\
\hline 3 & 3,5 & 3 & 2 & 1,5 & 1 \\
\hline 4 & 5 & 6 & 2.5 & 2 & 0,97 \\
\hline 5 & 6,5 & 7 & 3 & 3 & 0,88 \\
\hline 6 & 8 & 9 & 4.5 & 5 & 1 \\
\hline 7 & 11 & 14 & 5.5 & 8 & 1 \\
\hline
\end{tabular}

Step 1: Calculation the CCR Efficiency

Step 2: Calculation of $\Delta_{m, q}^{e f}$ 
DMU 1: $\Delta_{D M U 1,0}^{e f}>0$ _ DMU 1: Efficient.

DMU 2: $\Delta_{D M U 2,1}^{e f}<0$ _ DMU 2: Inefficient.

DMU 3: $\Delta_{D M U 3,1}^{e f}>0$ _ DMU 3: Efficient.

DMU 4: $\Delta_{D M U 4,3}^{e f}<0 \quad$ _ DMU 4: Inefficient.

DMU 5: $\Delta_{D M U 5,3}^{e f}<0$ _ DMU 5: Inefficient.

DMU 6: $\Delta_{D M U 6,3}^{e f}<0 \quad$ _ DMU 6: Inefficient.

DMU 7: $\Delta_{D M U 7,3}^{e f}<0 \quad$ _ DMU 7: Inefficient.

Solution of FConc algorithm: As DMUs 2, 4, 5, 6 e 7 are considered as INEFFICIENT and DMUs 1 e 3 are considered EFFICIENT.

\subsection{Comparative Analysis of the FConc and BCC Results}

As intended by its design, the FConc model generates an efficient frontier on which all efficient DMUs present increasing marginal productivity. Therefore, the returns generated by this model are global increasing returns to scale. Recall that the DEA BCC model presents efficient DMUs with decreasing marginal productivity, and so the outputs have decreasing global returns to scale. Note that the DMUs considered efficient in the FConc and BCC models are not the same, as the format of the frontier influences the result. In Example 1 and 2, the FConc model considered efficient DMUs that the BCC model had not considered. These DMUs that were neglected by the $\mathrm{BCC}$ can be considered efficient from the perspective of variable returns to scale, as they represent an increase in productivity. The inverse case can be observed in Figure 5, where some efficient DMUs in the BCC model were neglected by FConc. This fact explains the complementarity of the models in analyzing variable returns to scale.

In comparison with the DEA CCR model, both the FConc model and the DEA BCC model identify a greater number of efficient DMUs. This is because both models "relax" the proportionality condition of the CCR, which allows DMUs to be verified as efficient even without presenting the virtual output/virtual input relation of the efficient CCR DMUs.

\subsection{Characteristics of FConc Algorithm}

The FConc model has several characteristics that should be highlighted clarify its performance and better define its scope of analysis.

- Restriction of number of inputs/outputs

As the proposed algorithm initially arranges the DMUs, the variable chosen for this arrangement must be one-dimensional to avoid any doubt about the ordering. The FConc algorithm arranges the inputs in ascending order, so if the production process involves more than one input, more 
than one sorting order would be possible. For this reason, the algorithm should only be applied to problems with one input.

It is important to note that the algorithm could be applied to several inputs if the output quantity was one. In other words, either the input or the output must be unique. This feature means that multidimensional frontiers are cannot yet be considered.

- Verifying whether the FConc frontier always precedes the first CCR efficient DMU

Marginal productivity is a comparative measure. Consequently, the algorithm rules are influenced by this characteristic. Considering that the CCR-efficient DMU is that with the highest efficiency (equal to one), all other DMUs will have $\Delta_{O, q}^{e f} \leq 0$, i.e., they will be classed as inefficient by FConc. This means that, from the point at which the algorithm checks the first CCR-efficient DMU, no other DMU will be considered as FConc efficient.

This model ratifies the most widespread production frontier format in the economic literature, where increasing global returns to scale are verified before the CCR efficient DMU, and decreasing global returns to scale are verified after the CCR-efficient DMU.

- The first DMU will always be efficient

Considering that the first DMU (the DMU with the lowest input value) will consider $E f_{C C R q}=0$, the FConc model will always class DMU1 as efficient. This is because the first DMU will always have $E f_{C C R}>0$. An analogous property appears in the BCC model.

Therefore, if DMU1 is the CCR-efficient DMU, the model will present DMU1 as the only efficient FConc DMU.

\section{CONCLUSIONS}

This study presented a new nonparametric algorithm, FConc, for the analysis of productive efficiency under increasing global returns to scale. For this, the proposed model generates an efficient concave frontier along which the concept of increasing global returns to scale refers to the constant pattern of marginal productivity growth.

Initially, as a way of substantiating this research, we demonstrated the different types of verified variable returns to scale and the associated frontier formats. It was shown that, in the DEA literature, variable returns are only considered from the perspective of local returns to scale, and therefore only the average productivity variation is analyzed. The simple use of local returns, as more commonly found in specialized studies, makes it impossible to characterize the technology and the relationship between the variable returns and the frontier format. For this reason, the analysis of variable returns, from the perspective of increasing global returns to scale, has been neglected in the DEA literature. Hence, when considering DMUs with increasing marginal productivity as efficient, the proposed FConc model selects low-input-value DMUs that do not have 
sufficient scale to reach the productivity levels achieved by high-input-value DMUs. Therefore, models not considering increasing global returns to scale impair the analysis of efficiency for companies with smaller productivity scales than the CCR-efficient DMU, as they are considered to be inefficient. In this regard, the importance of this research lies in the delimitation of the DEA BCC model's scope of action and our demonstration that DEA BCC cannot identify DMUs with productivity scales smaller than the CCR-efficient DMU and presenting increasing marginal productivity.

The proposed FConc algorithm calculates the variation of CCR efficiencies between the studied DMUs and identifies whether there was an increase. The output of the proposed model is the DMUs belonging to the efficient frontier with increasing global returns to scale. All DMUs identified as efficient exhibit increasing marginal productivity, and the efficient frontier displays concave concavity.

Presenting the global returns to scale concept as an alternative definition of local returns to scale was the main motivation for conducting this research. The analysis of returns to scale under the global perspective showed that the DEA BCC model, which produces an efficient convex frontier, analyzes the productive efficiency of the DMUs under decreasing returns. Therefore, the analysis of globally increasing returns, as proposed in this study, is complementary and enriches the DEA literature.

A future challenge in this direction will be the definition of a multidimensional frontier, which would be useful for extending the applicability of the FConc model. Additional research toward this objective is important because, as already highlighted, the measurement of FConc model inefficiency is still limited to single input or single output examples. In this respect, the possibility of developing an FConc linear programming problem to measure FConc inefficiency in the case of $n$ inputs or $n$ outputs would generalize the application of the FConc model, both to determine efficient DMUs and to measure efficiency.

\section{References}

[1] Angulo Meza L, Biondi Neto L, Soares De Mello JCCB, Gomes EG \& Coelho PHG. 2005. Free software for Decision Analysis a software package for Data Envelopment models. In: ICEIS 2005 - Proceedings of the 7th International Conference on Enterprise Information Systems, pp. 207-212.

[2] Banker RD, CoOper WW, Seiford LM, Thrall RM \& ZhU J. 2004. Returns to Scale in Different DEA models. European Journal of Operational Research, 154: 345362.

[3] Banker RD, Charnes A \& Cooper WW. 1984. Some Models for Estimating Technical and Scale Inefficiencies in Data Envelopment Analysis. Management Science, 30(9): 1078-1092. 
[4] BANker R \& Thrall R. 1992. Estimation of Returns to Scale Using Data Envelopment Analysis. European Journal of Operational Research, 62: 74-84.

[5] Baumol WJ, Panzar JC \& Willig RD. 1982. Contestable Markets. Harcourt Brace Jovanovich, New York.

[6] Benicio J \& SoARes de Mello JC. 2015. Productivity analysis and variable returns of scale: DEA efficiency frontier interpretation. Procedia Computer Science, 55: 341-349.

[7] Benicio J \& Soares de Mello JC. 2019. Different Types of Return to Scale in DEA. Pesquisa Operacional, 39(2): 245-260. doi: 10.1590/0101-7438.2019.039.02.0245.

[8] Benicio J, Soares de Mello JC \& Meza LA. 2015. Efficiency in increasing returns of scale frontier. Operations Research and Big Data: IO2015-XVII Congress of Portuguese Association of Operational Research (APDIO), 15: 15-22. doi: 10.1007/978-3-319-24154-8_3.

[9] Charnes A, Cooper WW \& Rhodes E. 1978. Measuring the Efficiency of Decision Making Units. European Journal of Operational Research, 2(6): 429-444.

[10] Charnes A, Cooper WW, Lewin AY \& Seiford LM. 1995. Data Envelopment Analysis: Theory, Methodology and Applications. Kluwer Academic Publishers, Boston.

[11] Coelli T \& Perelman S. 1999. A Comparison of Parametric and Non-parametric Distance Functions: With Application to European Railways. European Journal of Operational Research, 117(2): 326-339,.

[12] Cooper WW, SeIford L \& Tone K. 2000. Data Envelopment Analysis: A Comprehensive Text with Models, Applications, References and DEA-Solver Software. Kluwer Academic Publishers, USA.

[13] Cooper WW, Shanling L, Seiford L, Tone K, Thrall R \& Zhu J. 2001. Sensitivity and Stability Analysis in DEA: Some Recent Developments. Journal of Productivity Analysis, 15(3): 217-246,

[14] Despotis DK, Stamati LV \& Smirlis YG. 2010. Data envelopment analysis with nonlinear virtual inputs and outputs. European Journal of Operational Research, 202(2): 604-613.

[15] Fried H, Knox Lovell C \& Schmidt S. 2008. The Measurement of Productive Efficiency and Productivity Growth. Oxford University Press.

[16] Miyazaki H. 2001. Scale Economies and Homogeneity. Microeconomics Lecture Supplements. Department of Economics, Ohio State University.

[17] Panzar JC \& Willig RD. 1977. Economics of Scale in Multioutput Production. Quarterly Journal of Economics, XLI: 481-493 
[18] Podinovski V. 2004. Local and Global Returns to Scale in Performance Measurement. The Journal of the Operational Research Society, Part Special Issue: Intelligent Management Systems in Operations 55(2): 170-178.

[19] QUINZII M. 1992. Increasing Returns and Efficiency. Oxford University Press.

[20] SEnRA LF et al. 2007. Estudo sobre métodos de seleção de variáveis em DEA. Pesquisa Operacional, 27(2): 191-207.

[21] Soares de Mello JC et al. 2005. Fronteiras DEA Difusas. Inv. Op., 25(1): 85-103.

[22] Soares de Mello JC, Angulo Meza L, Gomes E \& Leta F. 2008. DEA Advanced Models for Geometric Evaluation of used Lathes. WSEAS Transactions on Systems, 7(5): 500-520.

[23] Sueyoshi T. 1999. DEA duality on returns to scale (RTS) in production and cost analyses: An occurrence of multiple solutions and differences between production-based and cost-based RTS estimates. Management Science, 45: 1593-1608.

[24] Thanassoulis E. 2001. Introduction to the Theory and applications in Data Envelopment Analysis: A Foundation Text with Integrated Software. Kluwer Academics Publishers, Boston.

\section{How to cite}

Benicio J \& Mello JCS. 2020. Algorithm modeling for constructing a concave efficient frontier. Pesquisa Operacional, 40: e224070. doi: 10.1590/0101-7438.2020.040.00224070. 